\title{
Dos tipos de construcción admirativa. Aproximación a las formas de codificar la sorpresa desde la perspectiva de la gramaticalización*
}

\author{
Two types of admiring construction. Approach to the ways of codifying surprise \\ from the grammaticalization perspective \\ Guillermo Soto ${ }^{1}$ y Felipe Hasler ${ }^{2}$ \\ ${ }^{1}$ Universidad de Chile, Santiago, Chile. Correo electrónico: gsoto@uchile.cl \\ ${ }^{2}$ CONICET, Argentina y Universidad de Chile, Santiago, Chile. \\ Correo electrónico: keche.felipe@gmail.com
}

La admiratividad ha sido definida como la categoría semántica correspondiente a la sorpresa que provoca en el hablante la información que transmite cierta proposición (DeLancey 1997, 2001). En otro trabajo (Soto y Hasler 2013), la admiratividad se ha caracterizado como una categoría subjetiva compuesta por dos eventualidades: la eventualidad relatada y la sorpresa que ésta provoca. En el presente trabajo, proponemos que hay dos formas generales de codificar la admiratividad: el modo Ser, que expresa léxicamente la adquisición sorpresiva de cierta información, empleando un verbo de cognición o percepción, y el modo Er, que expresa léxicamente la eventualidad relatada y marca gramaticalmente la sorpresa que ésta genera en el hablante. Sólo en el segundo caso hay una categoría gramatical de admirativo.

Palabras clave: admiratividad, subjetividad, dominio epistémico, tipología lingüística

Mirativity has been defined as the semantic category dealing with the surprise provoked in the speaker by the information a proposition conveys (DeLancey 1997, 2001). In other work (Soto y Hasler 2013) mirativity has been characterized as a subjective category constituted by two eventualities: the narrated eventuality and the surprise provoked by this eventuality. In the present work, we propose that there are two primary ways in which mirativity is codified: the Ser way, that expresses lexically the unexpected acquisition of some information using a verb of perception or cognition, and the Er way, that expresses lexically the narrated eventuality and marks grammatically the surprise it provokes in the speaker or the listener. There is a grammatical admirative category only in the latter case.

Key words: mirativity, subjectivity, epistemic domain, linguistic tipology

El presente trabajo ha sido financiado por el proyecto SOC 10/19-2 de la Universidad de Chile. 


\section{INTRODUCCIÓN}

La admiratividad ${ }^{1}$ puede definirse como la categoría semántico-pragmática referida a la sorpresa que provoca en el hablante la información expresada por una proposición determinada (DeLancey 1997, 2001) ${ }^{2}$. Aunque es posible que la admiratividad se gramaticalice en un morfema propio, como es el caso de la terminación -ne del coreano (y, alternativamente, -kun (Aikhenvald 2004)), por lo general se expresa a través de diversas estrategias que pueden ir desde el empleo admirativo de morfemas de otro sistema, como ocurre con el morfema evidencial -rke del mapudungun (Hasler 2012), hasta su manifestación en construcciones o usos específicos, como sucede con el empleo del pretérito pluscuamperfecto en contextos ilocutivos admirativos del tipo ¡Habías sido tú! en español (Soto y Olguín 2010; Blestel 2011), una lengua que, al menos en su variedad estándar, carece de admirativos gramaticalizados. En este sentido, puede presentar diversos grados o niveles de convencionalización, sin llegar, necesariamente, a constituirse como categoría gramatical estricta en una lengua. En otro trabajo (Soto y Hasler 2013), hemos propuesto que se manifiesta en unidades lingüísticas de corte subjetivo (Langacker 1987, 2000), compuestas por dos eventualidades: una relacionada con la eventualidad relatada ${ }^{3}$, y otra con la sorpresa que ésta provoca.

En el presente trabajo planteamos que hay dos formas de expresar lingüísticamente admiratividad: un primer tipo, que hemos denominado 'Ser', que explicita léxicamente la adquisición sorpresiva del conocimiento de cierto fenómeno por el hablante, empleando un verbo de percepción o cognición (p. ej.: ver, darse cuenta); y un segundo, que hemos denominado ' $E r^{\text {'4 }}$, que agrupa aquellas construcciones que introducen de manera explícita la eventualidad relatada (i.e.: el conocimiento adquirido), marcando gramaticalmente la sorpresa que éste genera para el hablante ${ }^{5}$. Aunque estas formas no necesariamente dan lugar a unidades gramaticales estrictas, su reconocimiento es importante aun en lenguas no admirativas, como el español, pues contribuyen a explicar fenómenos de cambio diacrónico, contacto lingüístico y variación diatópica. Las dos formas pueden entenderse como dos polos de un continuo que puede avanzar desde las construcciones $\mathrm{Ser}$ a las $\mathrm{Er}$; hipótesis que se ilustra, en el presente trabajo, con ejemplos de la evolución diacrónica del quechua y el aymara, el español paraguayo en contacto con el guaraní y el español rural del sur de Chile. En tanto sólo las construcciones Ser

Siguiendo el criterio de Dik (1997), denominamos 'admiratividad' al dominio de significado potencialmente codificable y 'admirativo' al significado ya codificado en una lengua. En consecuencia, una lengua puede comunicar admiratividad a través de construcciones específicas sin que necesariamente haya unidades gramaticales estrictamente admirativas.

2 Aunque la mayor parte de la bibliografía ha restringido la codificación gramatical de la admiratividad a los casos en que ésta expresa convencionalmente la sorpresa del hablante, Hengeveld y Olbertz (2012) han propuesto una definición menos restrictiva, que incluye la sorpresa del oyente. En tanto la argumentación de Hengeveld y Olbertz (2012) es discutible, en el presente trabajo se mantiene la definición original de la categoría. Con todo, la validez de nuestra propuesta es independiente de la posición que se adopte respecto de la existencia de admirativas que codifican la sorpresa del oyente.

3 Usamos relatado en el sentido en que Jakobson (1975) habla de hecho relatado en su caracterización de los conmutadores en el sistema verbal.

4 Las abreviaturas responden al evento introducido léxicamente por la construcción. Así, Ser es la abreviatura para 'Sorpresa ante la adquisición de la eventualidad relatada' y $E r$ es la abreviatura para 'Eventualidad relatada'.

5 De ahí el empleo de las siglas Er y Ser: (sorpresa ante la) eventualidad relatada. 
marcan léxicamente la adquisición sorpresiva, este avance corresponde a un proceso de gramaticalización de la admiratividad. En otras palabras, no sostenemos que ambos tipos de construcción tengan el mismo status gramatical en el sistema: se trata de dos mecanismos distintos de expresión de una categoría semántico-pragmática, uno léxico y discursivo (Ser) y el otro gramatical (Er).

La exposición se organiza de la siguiente manera: en primer lugar, se caracteriza brevemente la admiratividad, relacionándola con dos categorías, la evidencialidad y el significado perfecto, que también portan un componente subjetivo y que desempeñan un papel importante en la caracterización de los tipos de admiratividad revisados en la presente investigación. Posteriormente, se exponen las principales características de las construcciones admirativas Er y se presentan ejemplos de lenguas amerindias de distintas familias: el mapudungun (aislada), el aymara (aymara), el quechua (quechua) y el guaraní paraguayo (tupí-guaraní). Más tarde, se presentan las principales características de las construcciones admirativas $\mathrm{Ser}$ con ejemplos del español. Finalmente, a modo de conclusión, se comparan ambos tipos de codificación o expresión y se presenta una breve aplicación de las propuestas presentadas al estudio, primero, del contacto lingüístico, a partir de la explicación de la construcción admirativa había sido -presente en el español paraguayo producto del contacto de dicha lengua con el guaraní (Blestel 2011)-; segundo, de la dimensión diacrónica del cambio lingüístico -a través de la explicación de la relación histórica que tienen los actuales marcadores de pasado no experimentado del quechua y el aymara con antiguas formas de tiempos compuestos-; y tercero, de la dimensión diatópica -a través de la explicación del uso del perfecto de subjuntivo más el morfema que para expresar admiratividad en zonas rurales de la VIII región de Chile-.

\title{
2. LA ADMIRATIVIDAD
}

La admiratividad es una categoría semántico-pragmática referida a la sorpresa que provoca en el hablante la información expresada por una proposición determinada (Delancey 1997, 2001). El siguiente ejemplo, del mapudungun, ilustra la categoría:

\author{
¡Müle-pa-rke-ymi am! \\ Estar-DIR-ADM-IND.2SG ${ }^{6}$ \\ ¡Así que estabas aquí! (yo no lo esperaba) (Hasler 2012: 176).
}

Se trata de una categoría relacionada, en términos amplios, con el dominio de lo epistémico, al vincularse con la adquisición no controlada del conocimiento de una situación que va en contra de las expectativas del hablante. En otro trabajo (Soto y Hasler 2013), hemos planteado que es posible caracterizar la admiratividad como una categoría con un componente (inter)subjetivo, compuesta por dos eventualidades: a) una eventualidad referida al hecho relatado (desde ahora $E r$ ), y b) una eventualidad

\footnotetext{
Las abreviaturas utilizadas en los ejemplos son las siguientes: $1=1$ persona; $2=2$ persona; $3=3$ persona; $\mathrm{AC}=$ Acusativo $\mathrm{ADM}=$ Admirativo $; \mathrm{CER}=$ Certeza $; \mathrm{CONT}=$ Continuativo $; \mathrm{DET}=$ Determinante DEM $=$ Demostrativo; DIR $=$ Direccional; DUB $=$ Dubitativo; EST $=$ Estativo; EVID $=$ Evidencial; EXP $=$ Experimentado; $\mathrm{HAB}=$ Habitualidad; IL = Ilativo; IND = Indicativo; NEG = Negación; $\mathrm{LOC}=$ Locativo $; \mathrm{OP}=$ Objeto Primario; PAS $=$ Pasado $;$ PART $=$ Participio $; \mathrm{PL}=$ Plural $; \mathrm{POS}=$ Posesivo $; \mathrm{PPOS}=$ Posposición $; \mathrm{PROG}=$ Progresivo $; \mathrm{NEXP}$ $=$ No Experimentado $; \mathrm{SG}=$ Singular; $\mathrm{TOP}=$ Topicalizador; $\mathrm{VAL}=$ Validador .
} 
referida a la respuesta sorpresiva al acceso a la información expresada en la primera (desde ahora Ser). Mientras Ser, como se puede observar en (2), pone en escena al conceptualizador, $E r$, ejemplificado en (3), mantiene el anclaje de la cláusula en la situación objetiva, por lo que consideramos que se trata de una construcción subjetiva atenuada o de primer grado (Langacker 2000).

(2) Nunca había visto respuestas más estúpidas (Soto y Olguín 2010: 90).

(3) Feymew kom-küle-ka-rke-y.

Entonces todo-EST-CONT-ADM-IND.[3SG]

Entonces resultó que seguía estando toda [la chicha]. (Salas 2006: 248).

Si bien se puede expresar de manera independiente, la admiratividad tiende a manifestarse mediante otros recursos, entre estos, notablemente, marcadores de evidencialidad, como en las oraciones (1) y (3) del mapudungun, donde se expresa a partir del morfema evidencial -rke, y de perfecto, como en el caso del español de las tierras altas de Ecuador (4):

(4) Me dieron penicilina a ver si era alérgico, y... he sido alérgico (Hengeveld y Olbertz 2012: 493).

En Soto y Hasler (2013) planteamos que esta recurrencia se explica básicamente por tres hechos interrelacionados, que vinculan el significado perfecto con la evidencialidad y la admiratividad: la presencia de dos eventualidades, la atenuación o subjetivización, y un significado epistémico en sentido amplio. Más específicamente, planteamos que, como proponen Nishiyama y Koenig (2010), el perfecto vincula dos eventualidades a partir de una evaluación epistémica del hablante. El hablante estima que, de cierta eventualidad $\mathrm{X}$ en un tiempo $t$, puede inferirse otra eventualidad $\mathrm{Y}$ en un tiempo t', posterior a t. Sugerentemente, una de las inferencias posibles, como plantea Dik (1997), es que el hablante ha accedido a la información X en t', lo que corresponde al significado de la evidencialidad. Ello parece explicar la deriva de marcadores de perfecto a evidenciales que se ha observado, por ejemplo, en el español americano andino, ejemplificada en (4) (Escobar 1997; De Granda 1999, 2001). Finalmente, en el admirativo se produce un desplazamiento desde la eventualidad del acceso a la información hacia la respuesta emotiva que provoca un tipo determinado de acceso en el hablante, lo que explicaría la conexión entre evidenciales y admirativos.

La caracterización propuesta sugiere un trayecto desde el contexto hacia el signo lingüístico, en otras palabras, de la pragmática a la semántica: mientras en el perfecto el grueso de la información es inferible y altamente dependiente del contexto, en el admirativo la relación ya se ha codificado: la construcción significa la reacción de sorpresa del hablante ante una información inesperada. Este proceso parece ser correlativo al de subjetivización langackareana: mientras el significado perfecto introduce una segunda eventualidad relacionada con el conceptualizador, en algún sentido inferible desde el contexto, el admirativo introduce directamente al conceptualizador, a través de poner en escena su respuesta emotiva ante la ocurrencia de la eventualidad relatada. 


\section{Dos TIPOS DE CONSTRUCCIONES ADMIRATIVAS}

\subsection{Introducción}

En el presente trabajo profundizamos en la idea de que las construcciones admirativas se encuentran compuestas por dos eventualidades, proponiendo que las distintas manifestaciones de esta categoría se pueden agrupar en dos tipos, de acuerdo con la manera en que se codifiquen (léxica o gramaticalmente) las eventualidades involucradas: el tipo Ser y el tipo Er. Por un lado, en las construcciones tipo Ser, se explicita léxicamente la adquisición sorpresiva del conocimiento de cierto fenómeno, por lo general por el hablante, empleando un verbo de percepción o cognición; en otras palabras, se introduce de manera explícita la eventualidad relacionada con el conceptualizador $(\mathrm{Ser})$, sin designar directamente la eventualidad relatada $(\mathrm{Er})$, que es recuperable del contexto. Por otro lado, en las construcciones tipo $E r$, propias de lenguas como el mapudungun o el aymara, se introduce léxicamente la eventualidad relatada $(E r)$ y se marca gramaticalmente la sorpresa del conceptualizador (Ser). En el presente apartado, se exponen las principales características de cada uno de los tipos, se presentan ejemplos de distintas lenguas no relacionadas para cada uno de ellos y, finalmente, se realiza una comparación de sus propiedades sintácticas y semánticas, enfatizando en las semejanzas que llevan a definirlas como construcciones admirativas, y en las diferencias que permiten su clasificación en dos tipos distintos, sólo uno de los cuales está codificado gramaticalmente.

\subsection{Construcciones Er}

En algunas lenguas amerindias de diferentes familias, la admiratividad se manifiesta en la eventualidad relatada. Se expresa a través de un sufijo que presenta, adicionalmente, un significado evidencial, como en mapudungun (aislada), o temporal-evidencial, como en quechua (quechua), aymara (aymara) y guaraní (tupíguaraní). Por ejemplo:

Mapudungun

(5)

$\begin{array}{lcc}\text { Wiño-me-rke-y } & \text { ta. } & \text { Andrea } \\ \text { Volver-DIR-ADM-IND.[3] } & \text { DET } & \text { Andrea } \\ \text { 'Se devolvió Andrea (antes de lo esperado)'. (Hasler 2012: 171). }\end{array}$

Aymara

(6) Jupa-x mäwisiklit(a) apa-ni-tay-na.

Él-TOP un bicicleta-(AC) traer-DIR-PAS.NEXP-IND.3SG

‘Él había traído una bicicleta'. (Quelca 2012: 594).

Quechua

(7) Kay-pi-(má) este-LOC-ADM ka-sha-sqaMaria-qa. ‘María está acá!’. (Faller 2002: 24).

Guarani

(8) O-ú-nipo-rae.

3SG-venir-PAS.VAL

'Verdaderamente vino'. (Palacios 1999: 61). 
En mapudungun, el morfema -rke no codifica sólo admiratividad sino también dos significados evidenciales: el reportativo y el inferencial (Hasler 2012), como se puede ver en los siguientes ejemplos:

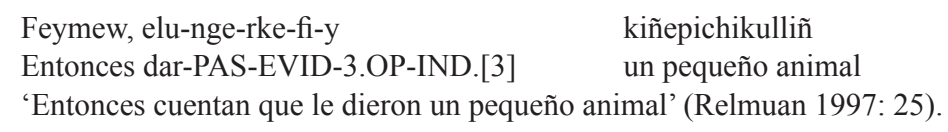

"urfi-pe-rke-la-y lafkenmewtachiweya pichi domo" ahogarse-CER-EVID-NEG-IND.[3] mar PPOS DET DEM pobre pequeña mujer fey fente-kintu-ke-rke-fi-y engün. Entonces terminar-buscar-HAB-EVID-3.OP-IND.[3] PL “"con seguridad se ha ahogado en el mar la pobre niñita" entonces hasta ahí no más la buscaron, cuentan'. (Salas 2006: 204).

Por su parte, el sistema temporal del quechua y del aymara distinguen dos tipos de pasados: el experimentado -expresado por -rqa en quechua y -ya en aymara-, y el no experimentado (o evidencial) -expresado por -sqa en quechua y -tay en aymara-. De acuerdo con Cerrón Palomino (2008), el pasado no experimentado expresa también acciones realizadas sin la participación voluntaria-consciente o no-del sujeto, por lo que es frecuente en las narraciones míticas y, además, en las acciones oníricas y en aquellas ejecutadas inconscientemente o recordadas por el hablante de su primera infancia. Como en el mapudungun, en estas lenguas el admirativo también se encuentra ligado a la evidencialidad, ya que se expresa a través de los sufijos de pasado no experimentado. Algunos ejemplos de los usos evidenciales de estos sufijos son los siguientes:

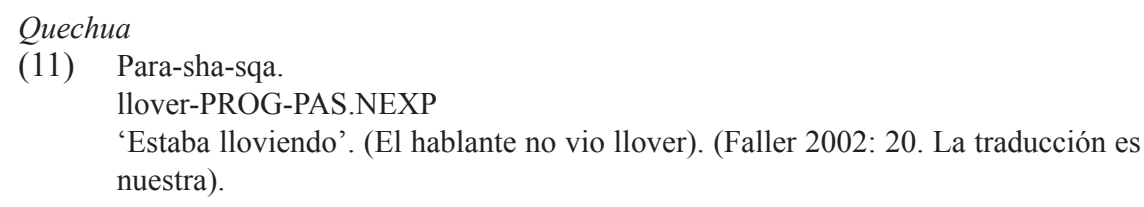

(12) Jupa-x jisk'akullaka-ja-ru-w nukt'a-tay-na Él-TOP ayer hermana-1.POS-IL-VALempujar-PAS.NEXP-IND.3SG 'Incluso ayer, le había empujado a mi hermanita'. (Quelca 2012: 593).

La interpretación del morfema -ra'e del guaraní ha generado mayor discusión. Palacios (1999: 61) señala que este sufijo expresa "pasado indefinido objetivamente en un tiempo no lejano al momento de la enunciación", por lo que se atribuye valor modal delegatorio, que no compromete al hablante con la verdad de su enunciado. Por ejemplo:
Peru o-ju-rae
che a-se-vo.
Pedro 3SG-venir-PAS.VAL
yo $1 \mathrm{SG}$-salir-cuando
‘Pedro vino cuando yo había salido'. (Palacios 1999: 61).

La autora señala, además, que puede usarse en combinación con validadores de duda (como ndaje) o de verosimilitud (como nipo), los que matizan o refuerzan el 
compromiso del hablante con su enunciado, como en:

(14) O-ú-ndaje-rae.

3SG-venir-DUB-PAS.VAL

'Dicen que vino'. (Palacios 1999: 61).

(15) O-ú-nipo-rae.

3SG-venir-CER-PAS.VAL

'Verdaderamente vino'. (Palacios 1999: 61).

Por su parte, Dietrich señala que este sufijo se utiliza mayoritariamente en preguntas y exclamaciones con referencia temporal pasada para expresar que el hablante no atestigua lo dicho, sino que tiene dudas o se maravilla de si fue posible lo que supone o pregunta (Dietrich 2009-2010: 78). De manera sugerentemente similar al quechua y al aymara, el sufijo -rae parece condensar significados temporales y evidenciales los que, en ciertos contextos, pueden extenderse hacia la expresión de la admiratividad (Blestel 2011).

Más allá de las diferencias formales en la codificación, en todas estas lenguas la eventualidad relatada se introduce como información nueva en el discurso, de manera explícita. Por otro lado, la respuesta del hablante ante ésta se introduce a partir de un sufijo que sólo indica la relevancia del tipo de acceso que el sujeto tuvo al conocimiento necesario para afirmar la eventualidad relatada, sin aportar información sintáctica adicional, esto es, sin introducir explícitamente ni un predicado ni una estructura argumental. En otras palabras, tanto el predicado como la estructura argumental de la construcción corresponden a la eventualidad relatada, la que se vincula con el hablante a través del sufijo de admiratividad. Dado que en todas estas lenguas el admirativo se codifica en conjunto con la evidencialidad -otro valor que porta un contenido epistémico-, el tipo específico de acceso debe ser precisado a partir de pistas contextuales, generalmente relacionadas con la ilocución.

\subsection{Construcciones Ser}

En este apartado revisaremos el uso admirativo del pluscuamperfecto español, observable en la construcción que ilustran los siguientes ejemplos:

(16) Nunca los había visto (Soto y Olguín 2010: 90).

(17) ¡No se me había ocurrido nunca! (Soto y Olguín 2010: 90).

Soto y Olguín (2010) plantean que esta construcción corresponde a un predicado que introduce dos eventualidades que contrastan entre sí: una eventualidad estativa 1, designada por la cláusula, en que el hablante no posee cierta propiedad epistémica $\mathrm{X}$, y una eventualidad estativa 2, que se sostiene en el momento de habla, en que el hablante posee dicha propiedad epistémica. Además, de manera implícita, se comunica que ha ocurrido un cambio, no controlado por el hablante, que ha ocasionado el paso desde la primera situación a la segunda: este cambio consiste, precisamente, en la adquisición del conocimiento X. Por lo tanto, el predicado en su conjunto corresponde a una situación dinámica, no controlada y télica -es decir, un logro vendleriano- que designa la adquisición o pérdida de conocimiento. 
Esta construcción se realiza a partir de un marco de predicación binario en que, por un lado, el primer argumento desempeña el papel de experimentante, designa al hablante y puede cumplir la función sintáctica de dativo, como en (18), o de sujeto, como en (19) y, por otro lado, el segundo argumento desempeña el papel de tema, designa el objeto de conocimiento y puede cumplir la función de objeto directo/ acusativo, como en (19), o de sujeto, como en (20). Ambos argumentos presentan, en los casos estudiados, información dada o conocida: en cuanto al argumento referido al hablante, al ser éste un participante del proceso enunciativo, su carácter de dado se asume por definición; por otra parte, el segundo argumento refiere a una información ya conocida por los interlocutores, lo que se manifiesta en el empleo de pronombres anafóricos y elipsis, como se puede observar en:

(18) No se me había ocurrido pensar en eso (Soto y Olguín 2010: 90).

(19) Ajaajajuajauajaua, no había leído eso (Soto y Olguín 2010: 91).

(20) ¡Se me había ido! (Soto y Olguín 2010: 90).

\section{Comparación ENTRE los TIPOS DE EXPRESIÓN DE ADMIRATIVIDAD}

Como se desprende de lo expuesto hasta aquí, ambos modos de expresión difieren en la gramaticalización de la sorpresa concomitante a la adquisición de cierta información. Mientras en un caso se lexicaliza el acceso a través de un verbo con marco predicativo; en el otro, el acceso sorpresivo se marca mediante un morfema. De ahí, pues, que sólo pueda hablarse de una categoría gramatical de admirativo en el segundo caso. Este contraste está a la base de cuatro diferencias semánticopragmáticas y sintácticas que se observan en las lenguas estudiadas, y que dicen relación con el estatus informativo, la integración clausal, el grado de dependencia contextual, y la incorporación del conceptualizador. Con respecto al primer punto, mientras en las lenguas Ser la cláusula introduce información conocida, recuperable discursivamente del contexto - de ahí el frecuente empleo de pronombres anafóricos y elipsis-, en las lenguas Er la cláusula introduce información nueva en el discurso. En cuanto a la integración clausal, mientras en las lenguas Ser predominan los verbos auxiliares, en las lenguas $E r$ predominan los sufijos, de lo que se infiere que en los segundos hay un mayor grado de integración clausal que en los primeros. Relacionado con lo anterior, mientras en las lenguas Ser el hablante se introduce explícitamente a través de mecanismos morfosintácticos -como la concordancia verbal o un pronombre dativo-, en las lenguas Er el hablante se introduce como una variable libre determinada contextualmente, por lo que es posible plantear, siguiendo a Langacker $(1987,2000)$, que en los segundos hay un mayor grado de incorporación subjetiva del conceptualizador, toda vez que existe una relación inversamente proporcional entre la cantidad de sintaxis que una forma necesita y el grado de significado subjetivo que expresa ${ }^{7}$. Finalmente, los elementos que dependen contextualmente también

Desde un punto de vista lingüístico-cognitivo, la cancelación sintáctica es una consecuencia natural de un proceso de subjetivización, ya que el hablante o conceptualizador que emite una construcción subjetiva no se enfoca en describir las entidades que tienen lugar en el mundo, sino que busca integrar su perspectiva del evento en la conceptualización. 
difieren en los dos tipos de construcciones. Mientras la dependencia del contexto en el caso de las construcciones $E r$ se reduce a la especificación de la forma de acceder al conocimiento (a través de la visión, de oídas, etc.), en las construcciones Ser la eventualidad relatada debe recuperarse del contexto, normalmente el discursivo.

\section{Aplicación al estudio de la dimensión diacróniCA, DiatóPICA y de CONTACTO ENTRE LENGUAS}

Del contraste en el estatus gramatical entre las construcciones $\mathrm{Er}$ y $\mathrm{Ser}$, se desprende que estas últimas podrían avanzar hacia las primeras en un proceso de gramaticalización. Como una manera de apoyar nuestros planteamientos, en el presente apartado presentamos dos casos en que esto parece ocurrir. El primero, correspondiente a un cambio motivado por el contacto lingüístico del español paraguayo con el guaraní, y el segundo, correspondiente, al parecer, a procesos de cambio diacrónico en quechua y aymara. Además, presentamos el caso de una variedad dialectal del español, hablada en zonas rurales de la VIII Región de Chile, en donde se registra el uso del perfecto del modo subjuntivo regido por el morfema que con valor admirativo. A diferencia de la construcción analizada en Soto y Olguín (2010), en este uso rural se observa, a nuestro juicio, un mayor avance en la gramaticalización $\operatorname{Ser}>E r$.

\subsection{Había sido en el español de Paraguay}

En el caso del español paraguayo, existe un uso adverbial del pretérito pluscuamperfecto del verbo ser que va en contra de las normas sintácticas del español (Blestel 2011). Este uso se puede observar en los siguientes ejemplos:

(21) Había sido se perdió su pasaporte, por eso no pudo viajar Marín. (Blestel 2011: 70).

(22) También le hicieron una nota al cantante Leo Barreto de "Los cucarachos del Paraguay", quien enseñó a vocalizar a Roque. Ellos había sido se reúnen siempre voi a tocar la guitarra y darle con todo al canto. (Blestel 2011: 70).

Siguiendo la interpretación de Blestel (2011), en estos casos los hablantes utilizan el pretérito pluscuamperfecto para expresar un significado admirativo. Ahora bien, como se puede observar a partir de los ejemplos, la construcción había sido presenta propiedades muy semejantes, o equivalentes, a las de las construcciones sufijadas de tipo Er, propio del guaraní. En efecto, en las construcciones en comento la eventualidad relatada se introduce léxicamente y la sorpresa del hablante se marca con una construcción fija y gramaticalizada en $3^{\mathrm{a}}$ persona, semejante, en este sentido, a un morfema, toda vez que no introduce un predicado ni posee estructura argumental. En otras palabras, en los ejemplos, había sido funciona como un operador admirativo con alcance sobre la cláusula que codifica la eventualidad relatada, tal y como ocurre en las lenguas tipo Er.

Pensamos que el guaraní motiva el cambio del pluscuamperfecto + 'ser' (habia sido), que pasa, de la expresión de una admiratividad tipo Ser, a la marcación gramatical de una de tipo $E r$, a partir de un proceso de subjetivización. Si nuestra 
interpretación es correcta, por la situación de contacto, había sido pasaría a funcionar como un operador admirativo adjunto a Er. Esto explicaría su pérdida de poder predicativo y estructura argumentativa, su integración en la cláusula que codifica la eventualidad relatada, y el enriquecimiento pragmático que permite incorporar la perspectiva del sujeto (Company 2004). Siguiendo a Cornillie (2007), consideramos que el fenómeno que comentamos obedece a una gramaticalización motivada cognitivamente, en la que las formas lingüísticas reciben un significado más abstracto y más orientado hacia el hablante.

\section{2. sqa y-tay en quechua y aymara}

Por otro lado, en el caso del quechua y el aymara, sugerentemente, Cerrón Palomino (2008) señala que los sufijos -sqa y -tay se construyen sobre la base de las formas participiales -sqa, del quechua, y -ta, del aymara. Desde un punto de vista histórico, indica el autor, en ambas lenguas estas formas son el resultado del ensamblaje de una forma verbal compleja compuesta por un verbo y su participio seguido del verbo 'ser' que era, efectivamente, el portador de las referencias personales y de la marca de tiempo pasado: -rqa, en quechua y -ya en aymara. Así, por ejemplo, la forma actual del quechua (23) provendría de (24), y la del aymara (25) provendría de (26):

\section{Quechua}

(23) puklla-sqa-ni. jugar-PAS.NEXP-IND.1SG

'Yo había jugado'. (Cerrón Palomino 2008: 142).

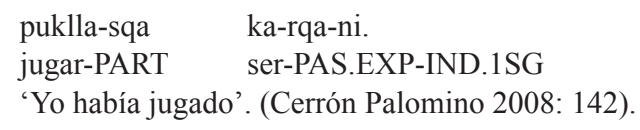

\section{Aymara}

(25) Anata-tay-tha. jugar-PAS.NEXP-IND.1SG

'Yo había jugado'. (Cerrón Palomino 2008: 142).

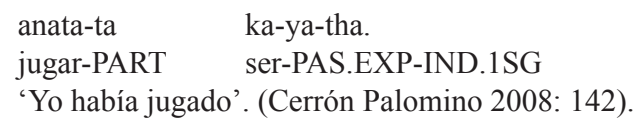

De acuerdo con esto, en un estado diacrónico anterior, tanto el quechua como el aymara contaban con un morfema de tiempo pasado, -rqa y -ya respectivamente, que no indicaba nada sobre la fuente de información del hablante. Junto con lo anterior, contaban con un tiempo compuesto formado por la cópula verbal en combinación con este morfema de tiempo pasado y el verbo de la construcción mezclado con una forma participial. En un estado diacrónico posterior, el sufijo participial pasó a marcar el significado de la construcción completa, obviándose tanto el uso de la cópula verbal como del morfema de tiempo pasado original. Además, pasó a formar una oposición basada en un parámetro evidencial -experimentado/no experimentado- con el morfema de tiempo pasado original y, además, adquirió matices de admiratividad en ciertos contextos. Si bien no poseemos datos suficientes como para afirmar el papel 
de la subjetivización en el proceso, resulta sugerente observar que el trayecto seguido por esta construcción en quechua y aymara replica lo planteado en nuestro marco teórico: de un tiempo compuesto, similar a los tiempos compuestos del español, a un significado evidencial, y desde éste a un significado admirativo.

\subsection{Haya + participio en el español rural de Chile}

Finalmente, en el caso del español de zonas rurales de la VIII región, hemos registrado los siguientes usos admirativos:

(27) ¡Y que lo haya mea[d]o el puma! (datos de campo ${ }^{8}$ ).

(28) ¡Que me haya robado la longaniza este gato hueón! (datos de campo).

(29) ¡Qué se haya tira[d]o este cabro! (datos de campo).

Los tres ejemplos provienen de relatos humorísticos referidos por sus protagonistas al entrevistador. El ejemplo (27) corresponde al final de un relato. El protagonista, víctima del puma, se estaba haciendo el muerto para no ser atacado por el animal. Luego de olfatearlo, el puma sorpresivamente levanta la pata, orina encima de él y se va caminando con indiferencia, generando el desenlace humorístico de la historia. El ejemplo (28) corresponde a un relato en el que el protagonista estaba cocinando una longaniza y, al descuidarse, entra un gato que se la lleva desde la sartén misma, lo que resultó muy sorpresivo para el narrador. Finalmente, el ejemplo (29) también se da al final de un relato. El protagonista le apuesta a un joven que no es capaz de lanzarse desde un puente de 5 metros de altura hacia el río que corre por debajo. El joven, en respuesta al desafío, va y se lanza, ante la sorpresa de los presentes. En los tres casos, la eventualidad relatada se introduce explícitamente, mientras que la sorpresa concomitante se introduce de manera implícita, con el uso del perfecto del subjuntivo, que permite al hablante anticipar la incredulidad del oyente y otorga a la construcción, en conjunto con el marco ilocutivo, el componente de contraexpectativa y admirativo.

\section{CONCLUSIÓN}

En el presente estudio hemos propuesto que la admiratividad, entendida como la categoría semántico-pragmática que refiere a la sorpresa que provoca en el hablante la eventualidad relatada por una proposición, puede expresarse por medio de construcciones de tipo Ser y de tipo Er. Mientras en las primeras se explicita léxicamente la adquisición sorpresiva del conocimiento de cierto fenómeno, utilizando un verbo de percepción o cognición, en las segundas se explicita léxicamente la eventualidad relatada, marcando gramaticalmente la sorpresa que ésta genera. Sólo en el segundo caso podemos hablar propiamente de una codificación gramatical de la admiratividad; en otras palabras, sólo lenguas que posean un morfema (o una categoría análoga) que codifique la sorpresa califican, a nuestro entender, como lenguas admirativas. No

8 Los ejemplos fueron extraídos de las notas de los investigadores, correspondientes al trabajo de campo realizado en la comuna de El Carmen (Provincia de Ñuble, VIII Región) en el mes de noviembre de 2012. Hasta donde 1lega nuestro conocimiento, este tipo de construcciones admirativas no ha sido registrada en la bibliografía sobre el tema. 
obstante, el reconocimiento de construcciones Ser parece relevante en la medida en que, a nuestro juicio, permite describir procesos de contacto, cambio diacrónico y, eventualmente, variación dialectal en que construcciones de tipo Ser pueden, vía un proceso de gramaticalización, derivar en operadores admirativos propiamente tales.

\section{OBRAS CITADAS}

Aikhenvald, Alexandra. 2004. Evidentiality. Oxford: Oxford University Press.

Blestel, Élodie. 2011. "El pluscuamperfecto de indicativo en contacto con tres lenguas amerindias". Lenguas Modernas 38.2: 63-82.

Cerrón Palomino, Rodolfo. 2008. Quechumara. Estructuras paralelas de las lenguas quechua y aymara. La Paz: CIPCA.

Company, Concepción. 2004. “¿Gramaticalización o desgramaticalización? El reanálisis y subjetivización de verbos como marcadores discursivos en la historia del español”. Revista de Filología Española 84.1: 29-66.

Cornillie, Bert. 2007. Epistemic modality and evidentiality in Spanish (semi)auxiliaries. A cognitive-functional approach. Berlin-New York: Mouton de Gruyter.

De Granda, Germán. 1999. "Retención hispánica y transferencia quechua en dos fenómenos morfosintácticos del español andino". Lexis 23.1: 137-152. .2001. Estudios de lingüística andina. Lima: PUCP.

DeLancey, Scott. 1997. "Mirativity: The grammatical marking of unexpected information". Linguistic Typology 1.1: 33-52. . 2001. "The mirative and evidentiality". Journal of Pragmatics 33.3:369-382.

Dietrich, Wolf. 2009-2010. "Sintaxis del guaraní chaqueño (chiriguano, tupí-guaraní). La cláusula y las relaciones interclausales". Amerindia 33-34: 333-363.

Dik, Simon. 1997. The theory of functional grammar. Part.1: The structure of the clause. Berlin-New York: Mouton de Gruyter.

Escobar, Anna María. 1997. "Contrastive and innovative uses of the present perfect and the preterit in Spanish in contact with Quechua". Hispania 80.4: 859-870.

Faller, Martina. 2002. Semantics and pragmatics of evidentials in Cuzco Quechua. Tesis doctoral. Stanford: Stanford University.

Hasler, Felipe. 2012. El sistema de evidencialidad en mapudungun y sus transferencias al español mapuchizado. Tesis para optar al grado de Magíster en Lingüística. Santiago: Universidad de Chile.

Hengeveld, Kees y Hella Olbertz. 2012. “Didn’t you know? Mirativity does exist!”. Linguistic Typology 16.3: 487-503.

Jakobson, Roman. 1975. Ensayos de lingüistica general. Barcelona: Seix Barral.

Langacker, Ronald. 1987. Foundations of cognitive grammar: Theoretical prerequisites. Stanford: Stanford University Press. .2000. Grammar and conceptualization. Berlin: Mouton de Gruyter.

Nishiyama, Atsuko y Jean Koenig. 2010. "What is a perfect state". Language 86.3: 611-646.

Palacios, Azucena. 1999. Introducción a la lengua y cultura guaranies. Valencia: Universitat de València.

Quelca, Heriberto. 2012. "El uso del verbo como conocimiento directo e indirecto en el castellano hablado de la ciudad de El Alto". Anales de la Reunión Anual de Etnología 23: 570: 610 .

Relmuan, María Angélica. 1997. Kiñeke nütram ka pentukun dungu feypiel pu Rapawe ka Rukapangui lof che. Temuco: UFRO.

Salas, Adalberto. 2006. El mapuche o araucano. Fonología, gramática y antología de cuentos. Santiago: Centro de Estudios Públicos. 
DOS TIPOS DE CONSTRUCCIÓN ADMIRATIVA. APROXIMACIÓN A LAS FORMAS DE CODIFICAR LA SORPRESA DESDE LA PERSPECTIVA DE LA GRAMATICALIZACIÓN

Soto, Guillermo y Nicolás Olguín. 2010. “№ se me había ocurrido nunca! Una construcción admirativa de pluscuamperfecto en español". Revista Onomázein 22: 83-105.

y Felipe Hasler. 2013. "Perfecto, antiperfecto, evidencial y admirativo: Decir un evento para comunicar dos". Círculo de Lingüística Aplicada a la Comunicación 54: 130-153. 
